\title{
Cardiac care: A look at the nursing responsibilities and care.
}

\section{Hajara Bashari*}

Nigerian Air Force Hospital, Lagos, Nigeria

\begin{abstract}
The heart is the engine room and major organ of the humans without which these beings cannot survive. The human heart is an organ that pumps and supplies blood round the body through the circulatory system. This is supplying oxygen and nutrients to tissues as well as removing carbon dioxide and other wastes.

Keywords: Cardiovascular system, Aorta, Epicardium.
\end{abstract}

Accepted on June 02, 2020

\section{Introduction}

The heart with issues is taken care medically by doctors, nurses and other medical practitioners. Cardiac Nursing is the specialty that deals and takes care of patients suffering from various conditions of the cardiovascular system and also has certain responsibilities on these patients such as postoperative care, stress test evaluation, monitoring and health assessments. The cardiac nurse plays a diverse role of not only caring for the patient but also educating the family on understanding the heart conditions and its treatment [1].

\section{Brief Anatomy and Functions of the Heart}

The heart is a muscular organ just like the size of one's first situated in the inner size to the chest between the lungs by the left size of every human being. The heart has an aorta and four chambers the atria and ventricles that is the upper and lower part of the heart. It has 3 layers epicardium, myocardium and endocardium.

It has two types of blood vessels the vein which brings oxygen free blood to and the artery which brings oxygen rich blood away from the heart [2].

\section{Causes of Heart Diseases}

This occurs when plaque develops in the blood vessel that lead to the heart which blocks oxygen and important nutrients getting to the heart. A plaque is a waxy substance made up of cholesterol, fatty molecules and minerals. This accumulates over a period of time causing high blood pressure through damaging or blocking the artery for example through cigarette smoking or high cholesterol level.

\section{Risk Factors for Heart Diseases}

Age women-55, Men 45

Obesity

Diabetes

Family history with heart problems

Physical inactive
Eating unhealthy diet

Clinical depression

Unhealthy lifestyle

Living sedentary life style

Excessive drinking

Staying in high stress environment without proper stress management technique.

Lack of proper management of diabetes.

Knowing the warning signs of heart diseases.

\section{Types of Cardiac Diseases}

There are different types of cardiac diseases that affects the structures or functions of the heart such for example:

Abnormal heart disease or arrhythmia.

Aorta disease.

Congenital heart disease.

Deep vein thrombosis and pulmonary embolism.

Heart attack or failure.

Stroke.

Vascular disease (blood vessel disease).

High blood pressure.

\section{Signs and Symptoms}

Pain or heaviness in the chest which is radiating to the left arm.

Indigestion, nausea, vomiting, upper abdominal or back pain sometimes with the shoulder.

Shortness of breath and sweating.

Fatigue and weakness.

Slowness in performing normal activities. 


\section{Treatment/Prevention}

Prevention by maintaining a healthy life style with routine exercises.

Maintain healthy diet.

Quit smoking.

Ensure to control high up, high cholesterol and diabetes.

Maintain a healthy weight.

Drink in moderation or stop.

Annual medical and physical exams.

\section{What is Cardiac Nursing}

Cardiac Nursing is one of the specialties of nursing that work with patients who have suffered from various conditions of the cardiovascular system.

\section{Who is a Cardiac Nurse}

A Cardiac Nurse is a Registered nurse who specializes in treating and caring for patients with different types of heart diseases or conditions [3].

\section{Skills to be Acquired by a Cardiac Nurse}

A cardiac nurse must have some basic skills to enable proper management of any client or patient with cardiac issues. These skills include [4-9]:

Basic life support

Advance cardiac life support

Electro Cardio Gram (ECG) is the use of an instrument to record the impulses generated as electricity that travels to the heart muscles through the use of electrodes on the skin.

Stress test that is monitoring the patient during exercise through the use of treadmill or bicycle.

Echo cardiograph is an ultrasound examination of the heart.

\section{Roles of Cardiac Nurse}

The cardiac nurse plays diverse roles by educating patients in preventive methods and treat patients in clinical settings, keeping records of their medical histories as well as performing diagnosis on the patients.

They speak and educate the patients" families and helping them understand various types of heart conditions. Educate them on relevant health problems, procedures, planning and assisting them in follow up care.

Must work with all ages, old, young as well as children.

Help patients recover at home through assessing and treating them.

Monitoring the cardiac and vascular readings regularly or as prescribed by the Cardiologist.
Monitoring stress test evaluation.

Providing the necessary care especially in cases of postoperative care.

Supporting patients changing life style gradually.

Generally, the roles of a cardiac nurse cannot be over emphasis and must also have the following

Customer service oriented.

Critical thinking.

Situational awareness.

Good judgement decision making.

Excellent communication.

Time management.

\section{Conclusion}

The heart is the engine room and major organ of the humans without which these beings cannot survive. The human heart is an organ that pumps and supplies blood round the body through the circulatory system by supplying oxygen and nutrients to tissues as well as removing carbon dioxide and other wastes. The heart with issues is taken care of medically by doctors, nurses and other medical practitioners. Cardiac Nursing is the specialty that deals and takes care of patients suffering from various conditions of the cardiovascular system. The cardiac nurse is a Registered nurse who specializes in treating and caring for patients with different types of heart diseases or conditions.

The roles and acquired skills of a cardiac nurse cannot be over emphasis. She plays diverse roles by educating patients in preventive methods and treat patients in clinical settings, keeping records of their medical histories as well as performing diagnosis on the patients. Educating the patients" families and helping them understand various types of heart conditions as well as assist them with follow up care. The nurse should be able to work with any age group and monitor the cardiovascular readings regularly. The nurse must be customer service oriented, have excellent communications and must be able to take good judgement decision. Maintaining a healthy life style is maintaining a healthy heart.

\section{References}

1. Stevanovic N. Anatomy and Physiology by Ross and Wilson. 2018.

2. AA Olowu JP. The Nurse as a Manager - Application of Management, Principles and Functions to Nursing (3rd edn). 2020

3. MedicineNet. 2020.

4. COVID-19 Antibody Tests: How Helpful Are They? 2020.

5. Chron. 2020.

6. Massetti M, Babatasi G, Rossi A, et al. Operation for atrial septal defect through a right anterolateral thoracotomy: current outcome. Ann Thorac Surg. 1996; 62(4):1100-03. 
7. Ma ZS, Dong MF, Yin QY, et al. Totally thoracoscopic repair of atrial septal defect without robotic assistance: a single-center experience. J Thorac Cardiovasc Surg. 2011; 141(6):1380-83.

8. Owais TA, Farber G, Garbade J, et al. Excision of a left atrial myxoma via a minimally-invasive technique: A possible routine access. Interactive CardioVascular and Thoracic Surgery. 2011; 12(5):875-77.

9. Poyrazoglu HH, Avsar MK, Demir S, et al. Atrial septal defect closure: comparison of vertical axillary minithoracotomy and median sternotomy. Korean J Thorac Cardiovasc Surg. 2013; 46(5):340-45.

\section{*Correspondence to}

Hajara Bashari

Nigerian Air Force Hospital

Lagos

Nigeria

E-mail: hajabash@yahoo.com 